INPLASY

PROTOCOL

To cite: Zhang. Prognostic factors or prediction models for POD24 in patients with

newly diagnosed FL: a

systematic review and metaanalysis. Inplasy protocol

202120034. doi:

10.37766/inplasy2021.2.0034

Received: 09 February 2021

Published: 09 February 2021

Corresponding author:

Huilai Zhang

huilai_zhang@163.com

Author Affiliation:

Department of Lymphoma, Tianjin Medical University

Cancer Institute and Hospital

Support: None.

Review Stage at time of this submission: Formal screening of search results against eligibility criteria.

Conflicts of interest:

None declared.

\section{Prognostic factors or prediction models for POD24 in patients with newly diagnosed FL: a systematic review and meta-analysis}

Zhang, $\mathrm{H}^{1}$.

Review question / Objective: - To explore the performance of current prediction models for progression of disease within 24 months(POD24) in patients with follicular lymphoma (FL) - To identify the potential risk factors for POD24 at diagnosis in patients with FL.

Condition being studied: Follicular lymphoma (FL) is the most common indolent lymphoma in the world with a median survival approaching 20 years. However, the course of the disease is extremely heterogeneous and therefore, at diagnosis it is difficult to predict survival. Roughly $20 \%$ of patients experience rapid progression of disease post first line treatment. The prognostic risk factors that could predict progression with 24 months (POD24) remains unclear.

INPLASY registration number: This protocol was registered with the International Platform of Registered Systematic Review and Meta-Analysis Protocols (INPLASY) on 09 February 2021 and was last updated on 09 February 2021 (registration number INPLASY202120034).

\section{INTRODUCTION}

Review question / Objective: - To explore the performance of current prediction models for progression of disease within 24 months(POD24) in patients with follicular lymphoma (FL) - To identify the potential risk factors for POD24 at diagnosis in patients with FL.

Condition being studied: Follicular Iymphoma (FL) is the most common indolent lymphoma in the world with a median survival approaching 20 years. 
However, the course of the disease is extremely heterogeneous and therefore, at diagnosis it is difficult to predict survival. Roughly $20 \%$ of patients experience rapid progression of disease post first line treatment. The prognostic risk factors that could predict progression with 24 months (POD24) remains unclear.

\section{METHODS}

Participant or population: Inclusion: adults with grade 1-3a follicular lymphoma, newly diagnosed without transform based on pathology, treated with first-line treatment. Exclusion: patients without follicular lymphoma, patients under 18 years of age, patients with grade $3 b$, studies with mixed populations (watch\&wait and first-line treatment), studies only included patients receiving 'watch \& wait' or who received rituximab or radiotherapy after diagnosis.

Intervention: Risk factors: any patient factor, or laboratory measure, molecular genetic factors that is measured in clinical practice at diagnose (or shortly after). Risk prediction model: any risk prediction model that was in development or validation including prognostic factors measured in clinical practice.

\section{Comparator: Not applicable.}

Study designs to be included: We included Prediction model development/validation studies, case control or cohort studies investigating the association between risk factors and interested outcomes. We excluded editorials, letters, case reports, and RCTs comparing the effect of two interventions without further analysis of risk factors for POD24.

Eligibility criteria: Studies with adults with grade 1-3a follicular lymphoma, newly diagnosed without transform based on pathology, treated with first-line treatment, that investigated risk factors of POD24.

Information sources: We plan to search Embase, Medline, and Cochrane library from the earliest records to October 2020 without limitations on language, scope countries, publication types, or time frame. We also plan to manually search any relevant sources.

Main outcome(s): POD24, which is defined as patients with first-line treatment who are refractory or progressed/ transformed/ relapsed within 24 months (=24 months) after diagnosis/ first-line treatment initiation/ randomization. The refractoriness, progression, transformation and relapse are defined by original studies.

Quality assessment / Risk of bias analysis: For studies investigating risk factors for relapse, we will used the QUIPS tool to assess the risk of bias of included studies. We will assess each study's risk of bias considering six domains: participation, attrition, prognostic factor measurement, confounding measurement and account, outcome measurement, and statistical analysis and reporting. For risk prediction models studies, we will use PROBAST to assess the five domains on risk of bias of prediction modelling studies: participant selection, outcome, predictors, sample size and flow, and analysis.

Strategy of data synthesis: Assessment of heterogeneity Heterogeneity will be characterized as clinical heterogeneity, methodological heterogeneity and statistical heterogeneity. Before performing meta-analysis, studies will be judged homogenous in terms of population characteristics, intervention and comparators employed, outcomes and antiviral cessation criteria, study design, and statistical metric. An 12 estimate greater than or equal to $50 \%$ accompanied by a statistically significant Chi2 statistic, will be interpreted as evidence of substantial levels of heterogeneity. We will explore the sources of heterogeneity and do subgroup analysis where possible. Measures of association For prediction model studies, we will use sensitivity, specificity, Area Under Curve (AUC) to measure the discrimination of a specific model. We will use the calibration statistics reported by original studies to summarize the calibration of a specific model. The prediction value of a specific model will be 
measured by relative risk (RR), odds ratios (OR), or hazard ratio (HR) with their $95 \%$ confidence interval (Cl). For risk factor studies, we will use RR, OR, or HR with their $95 \% \mathrm{Cl}$ to measure the relationship between risk factors and POD24. Statistical analysis For prediction models studies, meta-analysis will only be performed when studies are judged homogenous in terms of population characteristics, prediction models, outcomes, statistical metric and study design. For risk factor studies, metaanalysis will only be performed when studies are judged homogenous in terms of population characteristics, risk factors, outcomes, statistical metric and study design. Otherwise, data will be synthesized narratively. We will use random-effect method for all analyses. We will conduct separate meta-analyses of RRs, ORs (at similar follow-up points) and HRs, and unadjusted and adequately adjusted results.

Subgroup analysis: Not applicable.

Sensitivity analysis: Not applicable.

Country(ies) involved: China.

Keywords: follicular lymphoma, prognostic factor, meta-analysis, prediction model, progression.

Contributions of each author:

Author 1 - Huilai Zhang.

Email: huilai_zhang@163.com 